\title{
TRANSTORNOS MENTAIS ORGÂNICOS EM UM AMBULATÓRIO DE SAÚDE MENTAL BRASILEIRO
}

| Leonardo dos Reis'; Sandra Pereira²; Lucilene Cardoso; ${ }^{3}$ Edilaine Gherardi-Donato ${ }^{4} \mid$

\section{RESUMO}

Este estudo objetivou verificar as características sociodemográficas e a necessidade de internação em unidade psiquiátrica dos pacientes diagnosticados com o transtorno mental orgânico em tratamento em um serviço ambulatorial de saúde mental. Os dados foram extraídos dos prontuários de todos os pacientes em tratamento no referido serviço. Comparou-se o perfil dos pacientes diagnosticados com transtornos orgânicos com o perfil geral de pacientes do local. Comparou-se ainda os percentuais de pacientes que já necessitaram de internação psiquiátrica com transtornos orgânicos e outros diagnósticos. Constatou-se que a maioria dos pacientes diagnosticados com o transtorno mental orgânico, são do gênero masculino, com média de idade de 56,72 anos, nível de escolaridade predominante ensino fundamental, e que $24 \%$ já necessitaram de internação. Definiu-se então o perfil do paciente com transtorno orgânico, em grande parte dos casos como sendo homem, de idade acima da verificada da média observada no serviço de saúde, de baixa escolaridade e que muitas vezes pode necessitar de hospitalização psiquiátrica.

PALAVRAS-CHAVE: delirium, demência, transtorno amnéstico e outros transtornos cognitivos; epidemiologia; saúde mental

\section{ABSTRACT}

We examined the sociodemographic characteristics and the need for hospitalization in psychiatric patients diagnosed with organic mental disorder under treatment in an outpatient mental health. Data were abstracted from medical records of all patients treated in that service. We compared the profile of patients diagnosed with organic disorders with the general profile of the local patients. We compared also the percentage of patients who have required psychiatric hospitalization with organic mental disorders and other diagnoses. It was found that most patients diagnosed with organic mental disorder were male, mean age of 56.72 years, the predominant level of education was basic education, and $24 \%$ have required hospitalization. Defined then the profile of patients with organic disorder, in most cases as a man, age verified above the average observed in the health service, with low education and that can often require psychiatric hospitalization.

\section{KEYWORDS: delirium, dementia, amnestic, cogni- tive disorders; epidemiology; mental health}

\section{INTRODUÇÃO}

Atualmente o sofrimento psíquico tornou-se mais evidente por adquirir proporções alarmantes. De acordo com a Organização Pan-Americana de Saúde - OPAS e a Organização Mundial de Saúde houve nas últimas décadas um crescente aumento da prevalência de transtornos mentais na população. Podemos entender o quanto essas doenças afetam a população mundial, pois se estima, conforme essas organizações, que atualmente há cerca de 450 milhões de pessoas que sofrem de transtornos mentais ou neurobiológicos. É importante ressaltar que os sofrimentos psíquicos representam quatro das dez principais causas de incapacitação em todo o mundo (Medeiros, 2005), integram o quadro de doenças crônicas não transmissíveis, se apresentam como um problema de saúde global e representa uma ameaça à saúde e o desenvolvimento humano. As cargas dessas doenças recaem especialmente sobre países de baixa e média renda (Volcan, Sousa \& Horta, 2003).

Projeções epidemiológicas referentes à saúde mental enfatizam a magnitude dos problemas mentais que tendem a aumentar nos próximos anos e os diferentes transtornos têm contribuído para contextualizar esse panorama. Neste trabalho abordou-se como tema os transtornos mentais orgânicos, os quais são constituídos pelas demências, transtornos relacionados a algum tipo de lesão ou disfunção cerebral, delirium e síndrome amnéstica (ambos não induzidos pelo álcool ou por substâncias psicoativas), sendo que estes dois últimos não serão abordados neste trabalho em razão de que no serviço de saúde mental, utilizado como campo de pesquisa, não foram encontrados pacientes portadores destes transtornos.

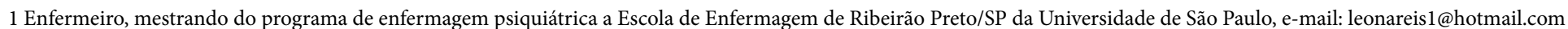

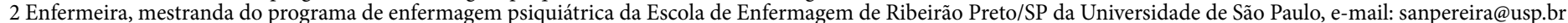

3 Prof. Dra. do Departamento de enfermagem psiquiátrica da Escola de Enfermagem de Ribeirão Preto/SP da Universidade de São Paulo, e-mail: lucilene@eerp.usp.br

4 Prof. Dra. do Departamento de enfermagem psiquiátrica da Escola de Enfermagem de Ribeirão Preto/SP da Universidade de São Paulo, e-mail: nane@eerp.usp.br 
As síndromes demenciais são caracterizadas pela perda de várias habilidades cognitivas e funcionais, por um empobrecimento progressivo dos processos psíquicos e afetivos e estão relacionadas a doenças como Alzheimer e Parkinson. Em geral, acometem a população mais idosa (Dalgalarrondo, 2000). Por tal razão, a maioria dos estudos encontrados à respeito, analisam populações de idosos. Estudo realizado no Brasil, em um serviço de emergência em saúde mental, com pessoas idosas, apontou os transtornos mentais orgânicos como a segunda causa mais frequente de atendimento (Almeida, 1999). Outro estudo, realizado em uma cidade do interior do estado de São Paulo, Brasil, com indivíduos acima de 65 anos de idade, apontou que mais de $7 \%$ dos entrevistados apresentavam algum tipo de demência (Herrera-Júnior, Caramelli \& Nitrini, 1998). Já os transtornos ocasionados por lesão cerebral, caracterizam-se por lesões em determinadas áreas do cérebro (frontal, temporal, áreas límbicas, núcleos de base, etc.) e ocasionam, na maioria das vezes, perturbações relacionadas ao humor, aos impulsos instintivos e à personalidade (Dalgalarrondo, 2000). Pesquisa realizada com idosos institucionalizados no Rio de Janeiro, Brasil, apontou que mais de $4 \%$ destes pacientes possuíam algum diagnóstico de transtorno mental relacionado à lesão ou disfunção cerebral (Hansel, Motta \& Silva, 2008). Outro achado importante, verificado entre pacientes com este tipo de transtorno, refere-se à relação entre a doença mental e a violência, sendo que cerca de $60 \%$ dos pacientes com lesões frontais apresentam comportamento violento (Valença \& Moraes, 2006). Com a implantação do Sistema Único de Saúde - SUS, lei 8.080/90, e com o andamento do processo de Reforma Psiquiátrica, lei 10.216/01, a sociedade brasileira conseguiu dar um grande passo para a conquista efetiva de um dos seus direitos sociais, a saúde, reconhecida pela Constituição Federal Brasileira de 1988 como "direito de todos e dever do Estado, garantido mediante políticas sociais e econômicas que visem à redução do risco de doença e de outros agravos e ao acesso universal e igualitário às ações e serviços para sua promoção, proteção e recuperação" (Constituição da Republica Federativa do Brasil, 1988). Estamos vivenciando um processo de transformações no modelo de assistência em saúde através da compreensão do processo saúde-doença e consequente prática de desinstitucionalização (Campos \& Soares, 2003). Por muitos anos a doença mental foi institucionalizada, trazendo consequências como de-socialização, negação da identidade, da subjetividade e perda de contratualidade. A Reforma Psiquiátrica surgiu para mudar o modelo assistencial, priorizando dispositivos de atenção à saúde mental extra-hospitalares de base comunitária (Paula, 2010). Para deter o fluxo de internações é necessário que o sistema ambulatorial seja eficiente, tenha resolutividade nos casos, impeça a internação e trabalhe pela ampliação de recursos intermediários entre o leito hospitalar e o ambulatório (Pezioli \& Moreira, 2007). Para o manejo do sofrimento psíquico não basta aplicação de técnicas, mas necessita de avanços na acessibilidade, na humanização e criação de estratégias de promoção à saúde que abordem de forma integral as questões sociais e de relacionamento interpessoal (Carvalho, Oliveira \& Rodrigues, 2010). As políticas públicas em saúde mental no Brasil dispõem sobre os direitos do indivíduo e redirecionam o modelo assistencial. É relevante que os estados e municípios alcancem uma política de saúde mental inclusa, equânime, extra-hospitalar, de base comunitária (Heck et al., 2008). É possível notar um aumento na demanda e ressaltar a importância dos serviços de níveis primários e secundários em saúde mental quando consideramos os esforços direcionados para a desinstitucionalização somados aos fatores de aumento da expectativa de vida da população. Este panorama facilita a observação de desafios a serem enfrentados por estes serviços, a fim de se adequarem e atenderem as necessidades de sua clientela de forma mais satisfatória. Uma maneira de se melhorar as ações do serviço corresponde em conhecer o perfil dos usuários, assim, torna-se possível direcionar ações mais específicas e apropriadas de acordo com o público atendido. Os princípios da equidade e integralidade merecem ser destacados, pois visam redução das desigualdades e garantia de fornecimento de um conjunto articulado e contínuo de ações e serviços preventivos, curativos e coletivos em todos os níveis de assistência. Para fornecer dados capazes de apontar tais especificidades entre o público atendido e também mostrar suas reais necessidades de saúde, evidencia-se a importância do levantamento epidemiológico como ferramenta na manutenção destes princípios (Scóz \& Fenili, 2003). O delineamento do perfil epidemiológico é importante para fornecer informações que possibilitem a realização de estudos de acordo com as características dos pacientes atendidos, independente dos serviços (Freitas, Maia e Iodes, 2006). Destaca-se ainda sua importância na produção de conhecimentos para a tomada de decisões frente à formulação de políticas de saúde, à organização do sistema e às intervenções destinadas a solução de problemas (Paim, 2003). Visando melhorar os serviços, tanto em termos de recursos humanos quanto físicos, torna-se indispensável planejar ações em saúde mental que visem identificar a real demanda correspondente a cada tipo de transtorno, suas peculiaridades, curso da doença, prejuízos sociais e tipo de tratamento. Instrumentos como SIAB (Sistemas de Informação da Atenção 
Básica) e SAI (Sistema de Informações Ambulatoriais), denominados pelo Ministério da Saúde do Brasil como instrumentos de gestão, visam orientar o planejamento e ações em saúde, pela tamanha importância conferida ao conhecimento epidemiológico (Almeida e Ferreira, 2008). Considerando a necessidade de um atendimento integral e equânime aos usuários de serviços de saúde mental, somado a falta de informações sobre o perfil epidemiológico da referida população e especialmente visando aumentar as chances de intervenções direcionadas aos pacientes com transtornos mentais orgânicos, tais fatores fomentaram a realização deste trabalho com o objetivo de conhecer as características sociodemográficas destes pacientes, bem como a relação destas com a necessidade de internação psiquiátrica.

\section{METODOLOGIA}

Projeto aprovado pelo comitê de ética em pesquisa da Escola de Enfermagem de Ribeirão Preto da Universidade de São Paulo, Brasil (protocolo 1446/2011), atendendo às normas estabelecidas pela Resolução 196/96 do Conselho Nacional de Saúde. Realizou-se um estudo quantitativo descritivo e exploratório, de natureza epidemiológica, de prevalência e correlacional. A amostra foi composta por todos os indivíduos em tratamento no Núcleo de Saúde Mental (NSM) do Centro de Saúde Escola da Faculdade de Medicina de Ribeirão Preto, Brasil, no período da coleta de dados, nos meses de abril e maio de 2012. As informações foram extraídas dos prontuários dos pacientes classificados como ativos no cadastro do serviço. O NSM é um serviço de natureza secundária e disponibiliza atendimento ambulatorial em saúde mental. No que se refere à análise dos dados, realizou-se a estatística descritiva das características sociodemográficas da população de pacientes, sendo consideradas as variáveis idade, em anos completos; sexo, dividido em masculino e feminino; diagnóstico, divididos conforme o capítulo V da CID-10 e nível de escolaridade, divididos nas categorias não alfabetizado, ensino fundamental incompleto, ensino fundamental, ensino médio incompleto, ensino médio, superior incompleto e superior. Descreveu-se ainda a variável denominada “internação", dividida em "sim", se o paciente já foi internado em unidade psiquiátrica pelo menos uma vez e "não", caso nunca tenha sido submetido a internação. Por fim, realizou-se a comparação das características dos pacientes diagnosticados com transtornos mentais orgânicos e o perfil geral do NSM. Sabe-se que o diagnóstico psiquiátrico depende de vários fatores, entre eles a forma de interpretação do profissional, e levandose em conta que o atendimento médico no NSM é feito por 3 psiquiatras diferentes, sendo que cerca de $93 \%$

50 | Revista Portuguesa de Enfermagem de Saúde Mental, 9 (JUN.2013) dos pacientes são divididos entre eles e os aproximadamente $7 \%$ restantes são atendidos por médicos residentes, foi admitido um viés relacionado à variável "diagnóstico" sendo aceito o diagnóstico mais recente constante no prontuário do paciente, independentemente do profissional responsável pelo seu tratamento (Dalgalarrondo, 2000).

\section{ANÁLISE DOS RESULTADOS}

Verificou-se que na ocasião da coleta de dados, havia 1281 pacientes em tratamento no NSM, destes, apenas 36 (aproximadamente 3\%) apresentaram como diagnóstico principal algum transtorno orgânico, dos quais, a maioria (mais de 86\%) apresentou o diagnóstico classificado pela CID-10 como F06 (outros transtornos mentais devidos a lesão e disfunção cerebral e doença física). Assim como observado no perfil geral do NSM, a maioria dos pacientes com transtornos orgânicos possui no máximo o ensino fundamental, no entanto apresentam maior percentual de indivíduos nesta categoria do que a população total do NSM, cerca de $86 \%$ contra quase $67 \%$. Quanto aos níveis secundários e terciários de instrução, observa-se o oposto o que denota que os pacientes com transtornos orgânicos, em geral, possuem menor nível de instrução se comparados ao perfil geral do NSM (gráfico 1).

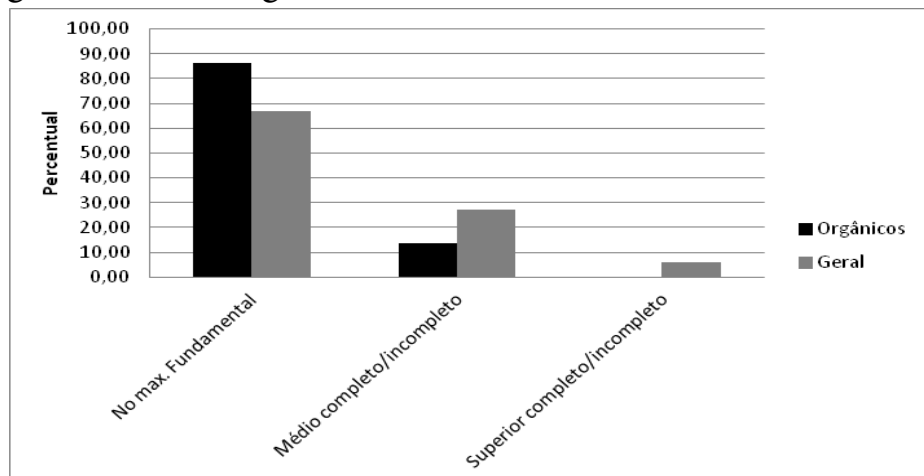

Gráfico 1 - Comparação entre as distribuições por nível de escolaridade, dos pacientes com transtornos orgânicos e quadro geral do NSM. Ribeirão Preto, 2012.

No que se refere à distribuição por gênero, verificou-se que no NSM as mulheres compõem a grande maioria (aproximadamente 69\%). Já em relação aos pacientes diagnosticados com transtornos orgânicos observa-se o oposto, sendo que os homens representam quase $60 \%$ do total (gráfico 2).

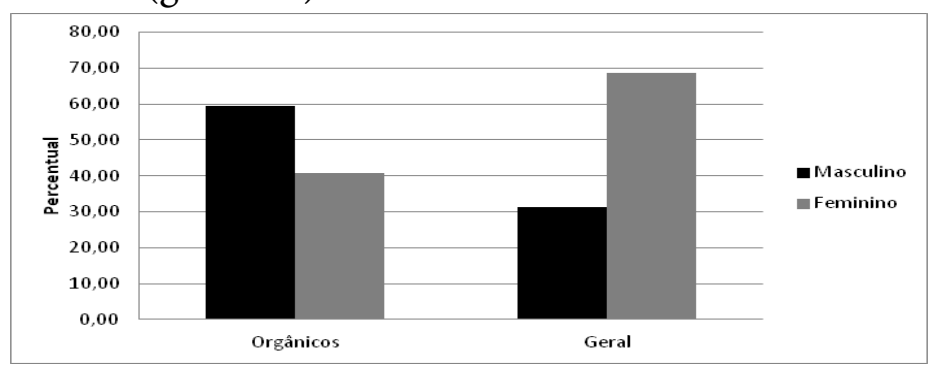

Gráfico 2 - Comparação entre as distribuições por gênero dos pacientes com transtornos orgânicos e quadro geral do NSM. Ribeirão Preto, 2012. 
Já em relação à distribuição etária, chama atenção, no que diz respeito aos pacientes com transtornos orgânicos, as faixas etárias de 30 a 49 anos (cerca de $46 \%$ dos pacientes) e acima de 70 anos (22\% dos pacientes). Se tomarmos como referência o perfil geral do NSM, estas faixas etárias representam aproximadamente $40 \%$ e $8 \%$ respectivamente (gráfico 3). Quanto à média de idade dos pacientes diagnosticados com transtornos orgânicos, apurou-se 56,72 anos, sendo o transtorno com maior média de idade do NSM. Já a média geral do NSM foi de 49,69 anos.

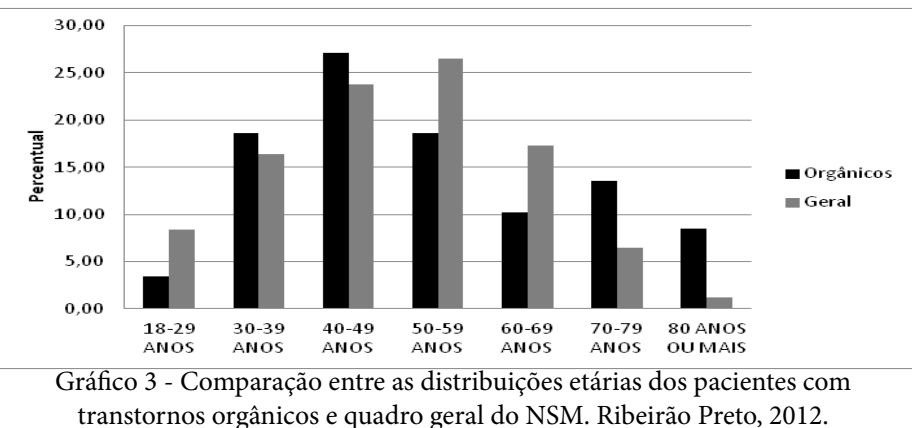

Em relação à necessidade de internação dos pacientes com transtornos orgânicos, o gráfico 4 apresenta o percentual de pacientes com este transtorno que já necessitaram de ao menos uma internação ao longo da vida e o percentual daqueles que nunca foram submetidos a hospitalização psiquiátrica. Para efeito de comparação, o mesmo gráfico exibe tais dados em relação a outros dois transtornos e ao perfil geral de pacientes do NSM. Quanto aos transtornos orgânicos, cerca de $24 \%$ dos pacientes com este diagnóstico já foram submetidos a internação psiquiátrica; percentual acima daqueles verificados no perfil geral do NSM (em torno de 18\%) e transtorno depressivo (aproximadamente 5\%), mas abaixo do observado para esquizofrenia (quase $37 \%$ ).

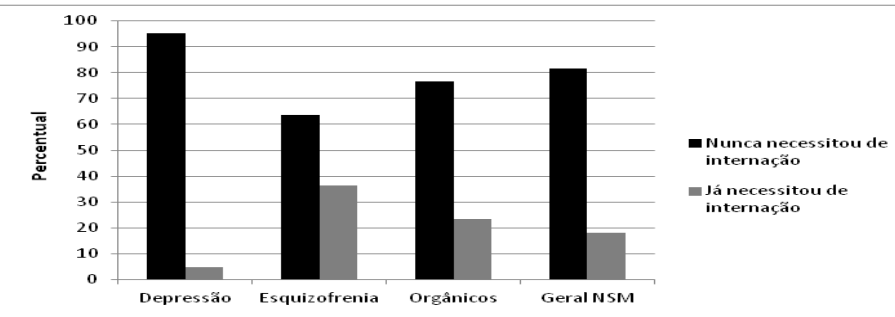

Gráfico 3 - Comparação entre percentual de indivíduos que já necessitaram de internação e percentual de indivíduos que nunca necessitaram de internação, segundo diagnóstico principal. Ribeirão Preto, 2012.

\section{DISCUSSÃO DOS RESULTADOS}

Estudos realizados no Brasil, em serviços semelhantes ao NSM, apuraram que os pacientes com diagnóstico de transtorno mental orgânico representam entre $4 \mathrm{e}$ $8 \%$ dos usuários destes serviços. Na cidade de Maringál PR foi onde se verificou o maior percentual (8\%) e em João Pessoa/PB o menor (4\%) (Carvalho, Oliveira e Rodrigues, 2010; Paula, 2010; Porcu et al., 2007). No NSM observou-se percentual pouco abaixo (3\%). Em relação a variável escolaridade, os achados apontam que, de uma forma geral, no NSM os pacientes com transtornos mentais orgânicos possuem nível de instrução abaixo da média geral dos pacientes. É sabido que o grau de instrução exerce forte influência sobre a capacidade de a pessoa interagir socialmente. Evidencia-se o fato de que a população de pacientes com transtornos mentais sofre discriminação relacionada à doença, o que de certa forma interfere no seu acesso à escola e consequentemente reflete em sua condição socioeconômica, tendo em vista que pesquisas apontam que quanto menor a escolaridade, menor a renda do indivíduo. Sabe-se também que a renda está fortemente associado à condição de saúde-doença das pessoas (Souza, 2007). A literatura demonstra que as pessoas com nível educacional inferior ao ensino fundamental tem escores mais altos para demência que os sujeitos com ensino médio ou superior, o que sugere que quanto mais baixo o nível educacional do indivíduo maior a possibilidade de ele apresentar transtorno mental (Souza, 2007). A maioria dos pacientes acometidos por transtornos mentais, neste estudo em especial aqueles com diagnóstico de transtorno mental orgânico, provavelmente não tiveram o acesso adequado à educação por conta de discriminações ou limitações relacionadas ao transtorno mental ou ainda por entraves causados pela condição econômica. No que se refere à variável gênero, verificou-se que entre aqueles com diagnóstico de Transtorno mental orgânico, a predominância é do gênero masculino (60\%), assim como no município de João Pessoa/PB, onde as observações apontaram que os homens representavam 59\% dos usuários (Medeiros, 2005). Diversos estudos tentam explicar as diferenças entre os gêneros relacionadas às manifestações dos transtornos mentais, com diferentes teorias e abordagens, no entanto, todos defendem a ideia do gênero como uma construção psicossocial que influenciará inevitavelmente a expressão da saúde mental (Rabasquinho e Pereira, 2007). Chama atenção em relação à distribuição etária o fato de que cerca de $46 \%$ dos pacientes diagnosticados com transtornos orgânicos estão dentro da faixa etária de 30 a 49 anos e $22 \%$ estão acima de 70 anos. Algo semelhante foi encontrado em um estudo realizado em uma cidade do nordeste brasileiro. Dos 2422 pacientes diagnosticados com transtornos mentais orgânicos, inclusive os sintomáticos, a maior concentração está na faixa etária de 20 a 39 anos (849 sujeitos), seguido por 761 pacientes na faixa etária de 40 a 59 anos e 454 pacientes acima de 60 anos (Medeiros, 2005). No município de Maringá grande parte dos pacientes com o mesmo diagnóstico encontra-se na faixa etária superior a 61 anos (Porcu et al., 2007). Estudo realizado em Campinas/SP, Brasil, 
mostrou que os pacientes com idade igual ou superior a 60 anos possuem 2,2 vezes mais risco de um desfecho menos favorável do que aqueles com menos de 60 anos no caso de necessitarem de internação psiquiátrica. Foi evidenciado ainda que os pacientes com diagnóstico de transtorno psicorgânico possuem, por exemplo, 2,7 vezes maior risco de um desfecho pior do que aqueles diagnosticados com transtornos de humor (Dalgalarrondo, Botega, \& Banzato, 2003). No NSM 24\% dos pacientes com transtornos orgânicos já necessitaram de ao menos uma internação durante a vida.

\section{CONCLUSÕES}

Em síntese, caracterizou-se o perfil dos usuários com transtornos mentais orgânicos como, em sua maioria, do gênero masculino, de baixa escolaridade e idade acima da média observada para outros diagnósticos. Muitos destes pacientes apresentam necessidade de internação psiquiátrica. Os estudos epidemiológicos em saúde mental visam não apenas o diagnóstico comunitário, mas o estudo do funcionamento do serviço, dos transtornos mais comuns na sua área de abrangência para assim direcionar as ações apropriadas de acordo com a população. Uma das formas de melhorar e aperfeiçoar a assistência consiste na caracterização da clientela assistida. Ressalta-se a importância de intensificar os cuidados ao portador de transtorno mental, através do cumprimento das políticas públicas de saúde e também ações no nível primário que atendam esta população prevenindo possíveis internações. Cabe à equipe preparar a família para lidar com este paciente, neste caso, especialmente aqueles acometidos por transtornos orgânicos, através de orientações quanto aos sintomas, evolução da doença e efeitos dos medicamentos.

Salienta-se que a taxa de atendimentos à população idosa tende a crescer devido ao envelhecimento da população. Assim, os serviços devem estar preparados para lidar com problemas de saúde característicos da terceira idade, como a demência e outros transtornos orgânicos, que apresentam prevalência aumentada em populações idosas. Espera-se que este estudo possa incitar ou servir de modelo para o uso de ferramentas de cunho epidemiológico no planejamento das ações e atenção aos pacientes além de melhorar a articulação da rede de saúde e melhor sistematização do cuidado.

\section{REFERÊNCIAS BIBLIOGRÁFICAS}

Almeida, G.C.M.; Ferreira, M.A.F. (2008). Saúde bucal no contexto do Programa Saúde da Família: práticas de prevenção orientadas ao indivíduo e ao coletivo. Cadernos de Saúde Pública. 24(9):21312140 .
Almeida, O.P. (1999). Idosos atendidos em serviço de emergência de saúde mental: características demográficas e clínicas. Revista Brasileira de Psiquiatria 21(1):12-18.

Campos, C.M.S.; Soares, C.B. (2003). A produção de serviços de saúde mental: a concepção de trabalhadores. Ciência \& Saúde coletiva. 8(2):621-628.

Carvalho, M.D.A.; Oliveira e Silva H.; Rodrigues, L.V. (2010). Perfil epidemiológico dos usuários da rede de saúde mental do município de Iguatu, CE. SMAD, Revista Eletrônica Saúde Mental Álcool e Drogas. 6(2):337-49.

Constituição da República Federativa do Brasil de 1988 (2010). Brasília. Recuperado em 15 de março de 2012 de http://www.planalto.gov.br/ccivil_03/constituicao/constitui\%C3\%A7ao.htm

Dalgalarrondo, P. (2000). Psicopatologia e semiologia dos transtornos mentais. Porto Alegre: artes médicas.

Dalgalarrondo, P.; Botega, N.J.; Banzato, C.E.M. (2003) Pacientes que se beneficiam de internação psiquiátrica em hospital geral. Revista de Saúde Pública. 37(5):629-34.

Freitas, R.M.; Maia, F.D.; Iodes, A.M.F. (2006). Atenção farmacêutica aos usuários do centro de atenção psicossocial - CAPS VI. Infarma. 18(9/10):12-16.

Hansel, C.G.; Motta, C.C.R.; Silva, J. (2008). Aplicação da escala de Katz (AVDs) na avaliação funcional de idosos com transtorno mental institucionalizados. In: $15^{\circ}$ Pesquisando em Enfermagem - Departamento de Enfermagem fundamental da Escola de Enfermagem Anna Nery/UFRJ. Recuperado em 15 de março de 2012, de www.pesquisando.eean.ufrj.br/viewabstract.php?id=461\&cf=2

Heck, R.M.; Bielemann, V.L.M.; Ceolin, L., Kantorski, L.P.; Wilhich, J.Q.; Chiavagatti, F.G (2008). Gestão e saúde mental: percepções a partir de um Centro de Atenção Psicossocial. Texto \& Contexto Enfermagem 17(4):647-55.

Herrera-Júnior, E.; Caramelli, P.; Nitrini, R. (1998). Estudo epidemiológico populacional de demência na cidade de Cantanduva estado de São Paulo. Revista de Psiquiatria Clínica 25(2):70-3.

Medeiros, E.N. (2005). Prevalência dos transtornos mentais e perfil socioeconômico dos usuários atendidos nos serviços de saúde em municípios paraibanos. Dissertação de Mestrado. Universidade Federal da Paraíba. Centro de Ciências da Saúde.

Organização Mundial de Saúde (OMS): Classificação Estatística Internacional de Doenças e Problemas Relacionados à Saúde - CID10. Genebra: 2008.

Paim, J.S. (2003). Epidemiologia e planejamento: a recomposição das práticas epidemiológicas na gestão do SUS. Ciência \& Saúde coletiva. 8(2):557-67.

Paula, C.T.C. (2010). Perfil epidemiológico dos usuários de um centro de atenção psicossocial na cidade de Recife. Cadernos Brasileiros de Saúde Mental. 2(4/5):94-105.

Pesioli, C.L.; Moreira, A.K. (2007). Avaliação de um centro de atenção psicossocial por meio do perfil de seus usuários. Mental. 5(8):61-75. 
Porcu, M., Previdelli, I.T.S.; Larini, M.C.F.; Mazaro, M.M.; Dias, T.G.C.; Oliveira, V.F. (2007). Prevalência dos transtornos mentais em pacientes atendidos no ambulatório de residência médica de psiquiatria da Universidade Estadual de Maringá. Acta Scientiarum. Health Science 29(2):145-149.

Rabasquinho, C.; Pereira, H. (2007). Gênero e saúde mental: Uma abordagem epidemiológica. Análise Psicológica. 3(25):439-454.

Scóz, T.M.X.; Fenili, R.M (2003). Como desenvolver projetos de atenção à saúde mental no programa de saúde da família. Revista Eletrônica de Enfermagem Recuperado em 15 de março de 2012 de https://www.revistas.ufg.br/index.php/fen/article/view/779/871.
Souza, A.R. (2007). Centro de atenção psicossocial: perfil epidemiológico dos usuários. 2007. Dissertação de Mestrado. Universidade Federal do Ceará. Faculdade de Farmácia, Odontologia e Enfermagem.

Valença, A.M.; Moraes, T.M. (2006). Relação entre homicídios e transtornos mentais. Revista Brasileira de Psiquiatria 28(2):62-8.

Volcan, S.M.A.; Sousa P.L.R.; Mari J.J.; Horta, B.L. (2003). Relação entre bem estar espiritual e transtornos psiquiátricos menores: estudo transversal. Revista de Saúde Pública. 37(4):440-5.

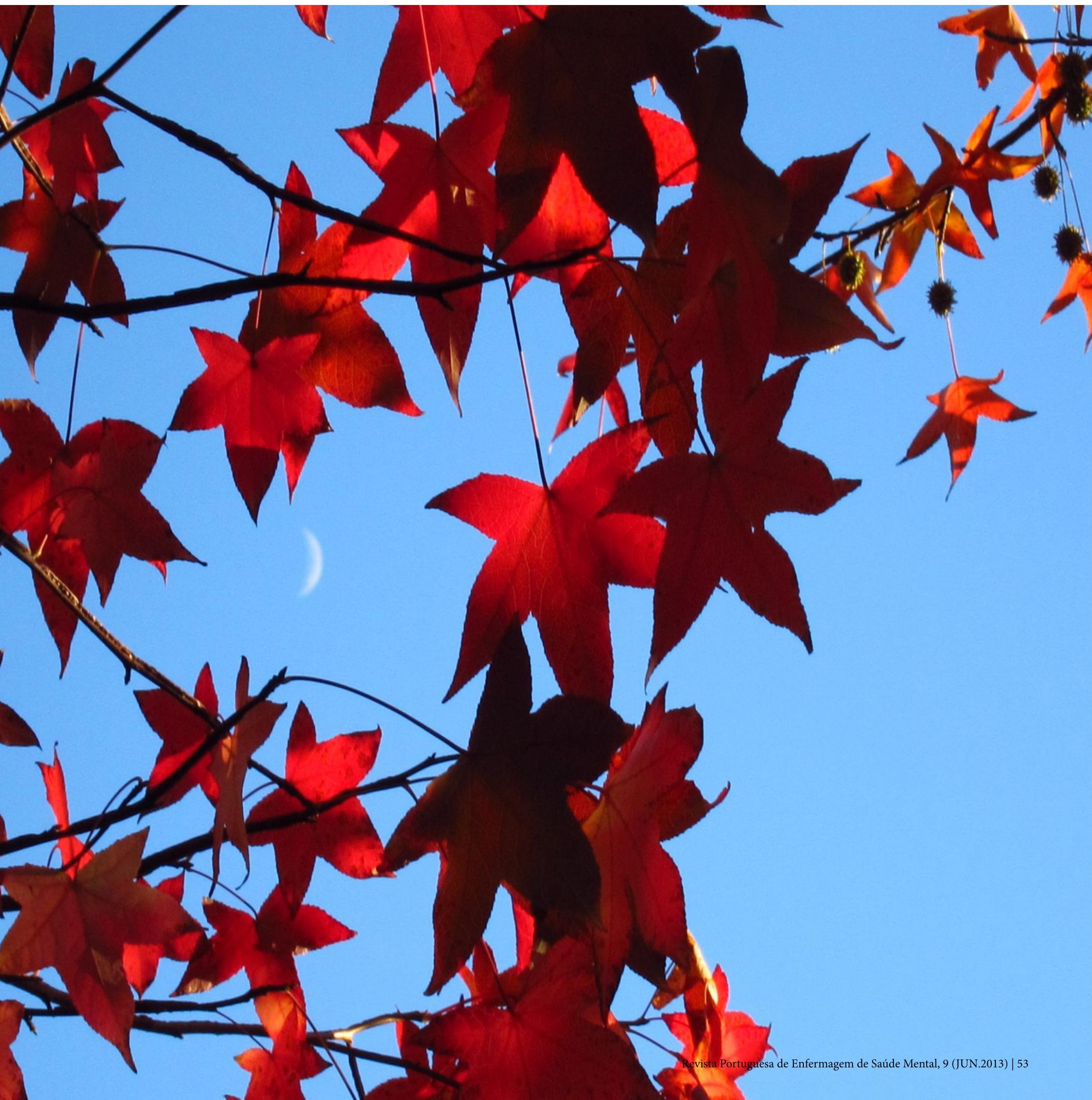

